\title{
Uso do acelerômetro e giroscópio no monitoramento de movimento: uma avaliação comparativa por meio de unidade inercial e smartphone
}

http://dx.doi.org/10.11606/1807-5509202000030429

\section{Resumo}

Este trabalho compara a aceleração mensurada por meio de sensores inerciais para monitorar a atividade física. 0 objetivo deste estudo é comparar informações sobre o movimento mensuradas por sensor inercial (sistema Xsens) e por um smartphone. Os dois instrumentos foram usados para medir a rotação de um pêndulo simples e da rotação da perna no movimento de agachamento. Para comparar o padrão temporal dos dados de rotação dos dois sistemas de medição, foi feita a correlação cruzada. Para estimar a rotação, foram usadas as medidas de quatérnion disponiveis em ambos os equipamentos. Para o pêndulo simples, as rotações descritas pelo quatérnions do sistema Xsens e do smartphone tiveram correlação cruzada muito forte, com semelhança de $98 \%$. No agachamento, o nível de semelhança de rotação dos quatérnions foi muita alta (97\%). 0 sensor de acelerômetro integrado ao smartphone apresenta alta semelhança a medida obtida por sensor inercial.

Palavras-chave: Atividade física; Biomecânica; Análise do movimento; Quatérnion.

\section{Introdução}

A análise do movimento e aplicação simultânea de métodos de mensuração denomina-se "Complexa investigação do movimento". Procedimentos eletrônicos são incorporados por meio de grandezas mecânicas, facilitando a medição que se altera rapidamente com o tempo, logo, essa transformação nos processamentos de dados permite mediçóes dinâmicas do movimento humano ${ }^{1}$. Entretanto, a interpretação dos dados de saída é complexa, o que restringe o entendimento das variáveis, direcionando a interpretaçôes subjetivas. A tecnologia facilita e oferece estratégias alternativas para interpretar as propriedades do padrão de movimento, porém, deve se insistir em estudos de forma a induzir a reprodutividade e evoluir em aspectos biomecânicos.

Acelerômetros têm proporcionado a descrição sobre os padróes de atividade física e comportamentos sedentários, o que permite associá-los ao nível de aptidão física e saúde das pessoas, ademais, verificar a eficácia de intervençôes para influenciar positivamente a prática de atividade física ${ }^{2}$ em diversos públicos.

É cada vez mais comum o uso smartphones para comunicação e transmissão de informação. Por causa disso, surge a oportunidade de integrar instrumentos de avaliação nos smartphones para obter informaçóes sobre o comportamento das pessoas. Os smartphones usam sistemas de localização e posicionamento para os aplicativos instalados em seu sistema operacional. Isso permite medir a aceleração linear e rotacional do aparelho, e a sua posição em relação ao campo gravitacional da Terra ${ }^{3}$. Por conter o acelerômetro e giroscópio triaxiais e o magnetômetro, o smartphone pode ser considerado como uma unidade de referência inercial (URI) ou sensor inercial. Portanto, é possível usar um smartphone para avaliar o equilíbrio postural de uma pessoa, pois o smartphone adquire com boa precisão e consistência os parâmetros de balanço corporal ${ }^{3}$.

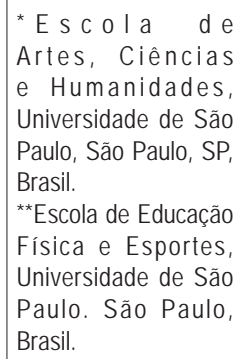


Para a avaliação do equilíbrio, o uso da smartphone apresentou informaçóes correlacionadas, obtida por meio da medida da trajetória do centro de pressão usando um sistema de avaliação do equilíbrio postural (NeuroCom, Biodex, EUA) ${ }^{3}$.

$\mathrm{O}$ sensor inercial instalado em equipamentos portáteis é a opção de estimativa não invasiva de movimento e pode ter aplicabilidade em diferentes cenários. Medir o movimento com precisão é essencial para avaliação clínica e acompanhamento da intervençáo terapêutica. Os sensores inerciais do sistema XSens podem ser usados para estimar o ângulo da articulação do joelho ${ }^{4}$. Para avaliação da condição de saúde, esse artefato pode monitorar atividades motoras grossas, e se tornar um indicador indireto de locomoção, tremor, gasto energético. Esse tipo de informação pode ser importante para confrontar com estados de diabetes, obesidade ou hipocolesterolemia; na progressão de doenças neurológicas que afetam a mobilidade; para estimar o risco de quedas ou detectar transições posturais ${ }^{5}$.

A representação do movimento do corpo humano no espaço requer informações de rotação, translação e orientação do corpo no espaço. As informaçóes disponíveis pelo sensor inercial permitem descrever a orientação e rotaçáo do corpo no espaço, porém, a medida de translaçáo do sensor inercial e o cálculo do deslocamento linear do instrumento no espaço são afetados pelo processo de dupla integração da aceleração linear, pois em cada integração é preciso estimar as constantes de integração (velocidade inicial e posição inicial). Como essa estimativa deve ocorrer em cada instante da mensuração, é comum ocorrer o acúmulo do erro das constantes e enviesar o cálculo numérico da translação. Para atenuar tal erro, é preciso adicionar à informaçáo do sensor inercial com dados externos para calibrálo em relaçáo ao ambiente. Isso limita a estimativa de medida de distâncias do sensor inercial sem as medidas externas do sistema de posicionamento global (global position system, GPS). Portanto, o smartphone tem potencial de uso como instrumento de medida do movimento de rotação e orientaçâo do corpo humano.

A rotação e orientação do corpo humano podem ser representadas em diferentes formas. A representação mais comum é por meio dos ângulos de rotação em eixos cartesianos que estão geralmente associados ao sistema de referências do ambiente, e os eixos representam a vertical e as horizontais. Para o corpo humano, por causa do movimento de cada articulação, é preciso usar como referencial cada segmento corporal e não a linha vertical, por exemplo. Desta forma, os ângulos de Euler são mais adequados para representar a rotaçáo de cada articulação. Ângulos de Euler permitem representar, também por meio de eixos ortogonais como os cartesianos, a rotação interna e externa, flexão e extensão, e abduçấo e adução de um segmento. Porém, os ângulos de Euler são influenciados pela ordem das rotaçóes em cada eixo e diferentes ordens de rotação geram diferentes posições finais. Por exemplo, começar movimento do ombro por rotação interna/externa ou flexão/extensão muda a posição final da mão. Logo, a ordem de rotaçōes dos ângulos de Euler afeta a orientação do segmento. Outro problema na representação da rotação por meio de ângulos de Euler é que quando a rotação em eixo coincide com a rotação em outro eixo, o número de graus de liberdade do sistema diminui, este problema é conhecido como "gimbal lock dos ângulos".

Alternativa para representar a rotação e orientação e superar o problema do "gimbal lock dos ângulos" de Euler e a limitação da representação por segmentos do sistema cartesiano é o quatérnion ${ }^{6}$. O quatérnion q é usado para representar a rotação e orientação de um objeto por meio de três variáveis vetoriais $\mathrm{x}$, y e z e uma variável escalar w. Estas três variáveis vetoriais representam a posição no espaço e a variável escalar representa o ângulo de rotação do sistema, como está apresentado a seguir:

$$
q=w+x \vec{i}+y \vec{j}+z \vec{i}
$$

\section{Equação 1}

Nos sensores inerciais de uma smartphone, as seguintes informaçóes são tipicamente apresentadas: aceleração linear nos três eixos ortogonais ( $\mathrm{X}$ - largura, Y - comprimento, e Z - espessura); aceleração angular em torno destes três eixos. Além disso, também é apresentado as variáveis do quatérnion. Existem poucos estudos sobre o uso de quatérnions na análise de movimentos para avaliação da atividade física, apesar de os quatérnions serem usados para robótica ${ }^{7}$ e mecânica de satélites ${ }^{8}$. Como a informação do quatérnion é menos suscetível a problemas de mensuração que os ângulos de Euler, será que a medida dos quatérnions de um smartphone pode ser usada para avaliar o movimento humano? Pretendemos comparar as 
informaçôes obtidas por um sensor inercial e por meio um smartphone. Para isso, o objetivo deste estudo é comparar as medidas de quatérnion de sistema de captura de movimento baseado em sensores inerciais e as medidas de quatérnion de um smartphone durante o movimento de agachamento. Se as medidas de quatérnion

\section{Métodos}

Foi usado um smartphone (Iphone 5, Apple Inc.) com as dimensóes $123,8 \mathrm{~mm}, 58,6 \mathrm{~mm} \mathrm{7,6} \mathrm{mm} \mathrm{e}$ massa $112 \mathrm{~g}$. Os sinais dos sensores de movimento (Giroscópio de três eixos, acelerômetro de três eixos, magnetômetro e sensor de proximidade ${ }^{9}$ ) do aparelho foram obtidos por meio do aplicativo Sensor Log v.1.8 (App Store by Bernd Thomas) ${ }^{10}$.

Foi um usado um sistema de sensores inerciais para avaliar o movimento. OXsens é um sistema de sensores inerciais que contém acelerômetros, giroscópios e MEMS (sensores de microeletromecânica) ${ }^{11 .}$

Os sinais foram sincronizados a partir da detecção do início do movimento em ambos os sensores. A correlação cruzada foi feita para obtenção de informações de rotação da central inercial, do smartphone em movimento controlado (balanço de um pêndulo simples) e do movimento dinâmico (agachamento sem carga externa). A frequência de aquisição dos dois sistemas foi $100 \mathrm{~Hz}$. Foi usado o aplicativo para IOS SensorLog e o software MT manager para Xsens.

\section{Protocolo}

A montagem dos instrumentos está apresentada na FIGURA 1. Foi construído um pêndulo simples com uma haste de metal de $20 \mathrm{~cm}$ de comprimento que será fixado a central inercial e ao smartphone. $\mathrm{O}$ raio do pêndulo de aproximadamente $12 \mathrm{~cm}$. Uma mulher ( 25 anos, 1,63 cm, $57 \mathrm{~kg}$ ) fisicamente ativa realizou os agachamentos simples. Foram feitos três blocos de três oscilações do pêndulo simples até a parada total e cinco séries de cinco agachamentos completos.

O sensor Xsens foi firmemente fixado ao smartphone (FIGURA 1). Na orientação vertical, a partir de uma impulsão manual, o pêndulo moverá cerca de $90^{\circ}$ no sentindo horário. A distância do ponto superior ao ponto fixo do sensor foi de $15 \mathrm{~cm}$. Em cada ensaio serão coletados três balanços consecutivos em forem semelhantes, o uso de smartphones pode ser ampliado para avaliar a orientação e rotação do corpo durante a prática de atividade física. Por ser um instrumento cada vez mais comum, isso permitirá a avaliação in loco do movimento de pessoas em programas de atividade física, treinamento e auto-avaliação.

orientação horizontal. A amplitude de aceleração utilizada foi de movimento humano, sendo configurada a amostragem entre 4-9 segundos.

Para o agachamento simples, o sensor foi calibrado no início dos ensaios. Os sensores foram fixados com ajuda de tiras. Dois sensores tiveram de ser posicionados no corpo do indivíduo (FIGURA 1), sendo um sensor colado ao smartphone e fixado na coxa Direita e o outro sensor alocado lateralmente a porção medial da tíbia. A referência para demarcação do ponto de fixação foi o vasto medial do quadríceps femoral ${ }^{12}$. De maneira sincronizada, ativou-se o aplicativo Sensor Log em conjunto a central inercial, e o sujeito foi convidado a realizar cinco séries de cinco agachamentos completos.

\section{Ambiente}

Os ensaios foram realizados no Laboratório de Biomecânica da Universidade do Estado de Santa Catarina (UDESC).

\section{Análise estatística}

Uma rotina no MatLab foi criada para analisar o padrão do movimento. O programa OriginPro8 foi utilizado para plotagem dos gráficos e identificação da variabilidade das variáveis. Foi calculado o índice de correlaçáo cruzada $\mathrm{R}$ entre as curvas dos quatérnions do celular e da central inercial. Além disso, foram calculados coeficientes de determinaçáo (R2). Para classificar a correlação, os seguintes valores foram usados ${ }^{13}: 0,9$ para mais ou para menos indica uma correlação muito forte; 0,7 a 0,9 positivo ou negativo indica uma correlaçáo forte; 0,5 a 0,7 positivo ou negativo indica uma correlaçâo moderada; 0,3 a 0,5 positivo ou negativo indica uma correlaçáo fraca; e 0 a 0,3 positivo ou negativo indica uma correlação desprezível. 
a)

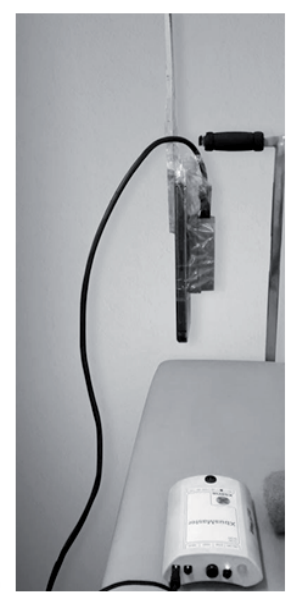

b)

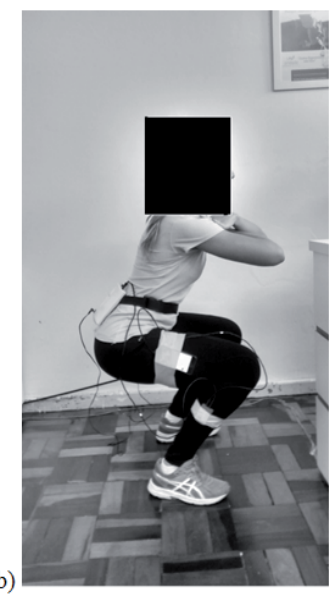

FIGURA 1 - Montagem experimental indicando o posicionamento dos sensores de medida Xsens e Iphone no a) pêndulo simples e no b) agachamento simples sem carga.

\section{Resultados}

As curvas temporais dos quatérnions estão representadas nas FIGURAS 2 e 3. Os valores dos índices de correlação cruzada entre as curvas dos quatérnions mensurados pelo smartphone e pela central inercial no teste do pêndulo simples estão apresentados na TABELA 1. Para a correlaçáo cruzada, os valores médios foram $0,991 \pm 0,005$ (quatérnion $\mathrm{x}$ ), 0,314 $\pm 0,244$ (quatérnion $\mathrm{y}$ ), $0,069 \pm 0,025$ (quatérnion z), 0,312 $\pm 0,242$ (quatérnion w $0,998 \pm 0,000$ ). $\mathrm{O}$ coeficiente de determinação R2 apresentou os valores $0,98 \pm 0,00$

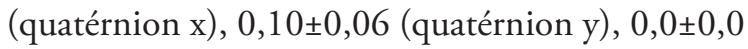

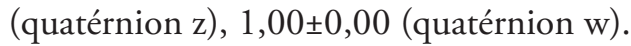

No agachamento foram identificados nos eixos $Z$, W e rotação, a semelhança de $97 \%$, 97\% e 100\%, respectivamente. A detecção angular de flexão e extensão dos joelhos também foi reconhecida com uma correlação muito forte de 92\% (TABELA 2).

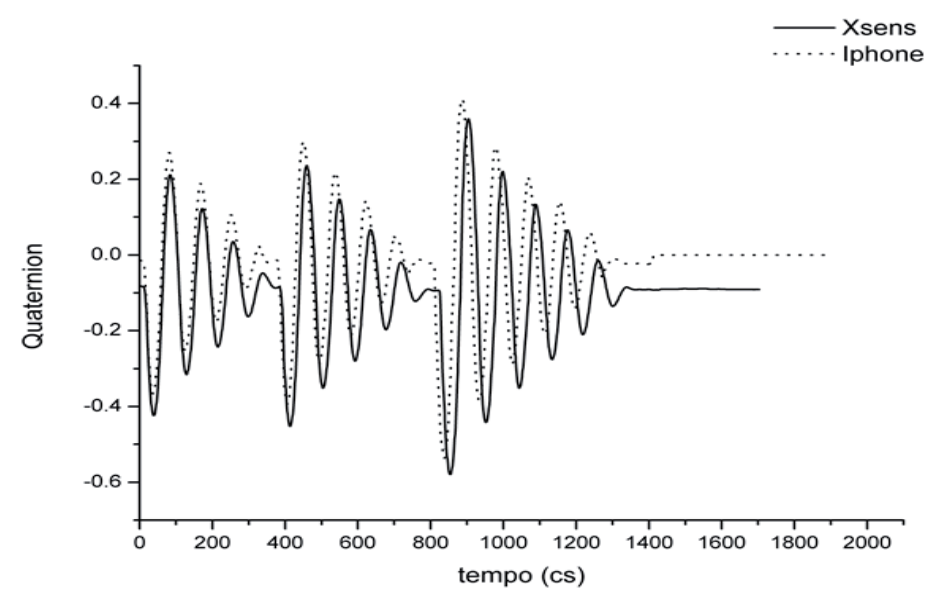

FIGURA 2 - Quatérnion representando a rotação do pêndulo simples mensurado pelo Xsens e Iphone. 


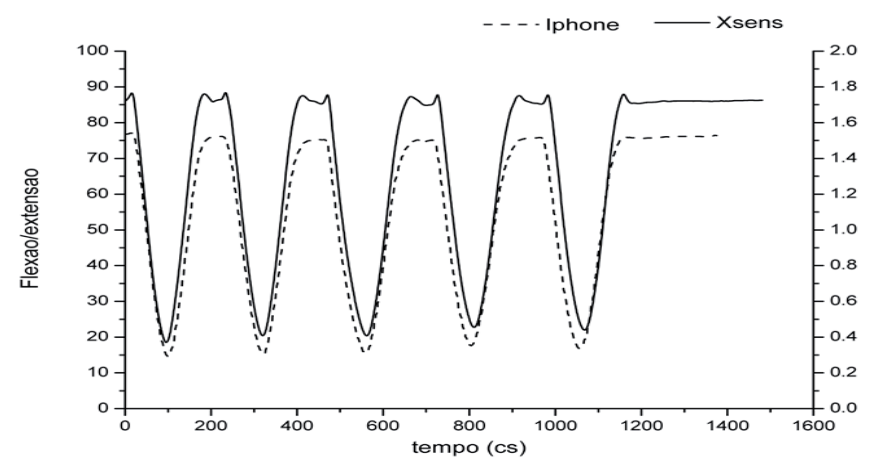

FIGURA 3 - Quatérnion representando a rotação (flexão e extensão) do segmento coxa durante o agachamento medido pelo Xsens e Iphone.

TABELA 1 - Valores médios e desvio padrão (DP) índice de correlação cruzada R da relação dos quatérnions medidos no celular e na central inercial no teste do pêndulo simples.

\begin{tabular}{lccccc}
\hline & \multicolumn{2}{c}{ Correlaçáa R } & & $\begin{array}{c}\text { Coeficiente de } \\
\text { Determinaçáo } \mathbf{R}^{2}\end{array}$ \\
\hline Variável & Teste 1 & Teste 2 & Teste 3 & Média(DP) & Média(DP) \\
\hline Quatérnion x & 0,991 & 0,996 & 0,987 & $0,991 \pm 0,005$ & $0,98 \pm 0,00$ \\
Quatérnion y & 0,333 & 0,548 & 0,060 & $0,314 \pm 0,244$ & $0,10 \pm 0,06$ \\
Quatérnion z & 0,046 & 0,068 & 0,095 & $0,069 \pm 0,025$ & $0,00 \pm 0,00$ \\
Quatérnion w & 0,998 & 0,998 & 0,998 & $0,998 \pm 0,000$ & $1,00 \pm 0,00$ \\
\hline
\end{tabular}

Os valores estão apresentados por teste $\mathrm{e}$ valor médio.

Quatérnion x é a rotação em torno do eixo perpendicular a tela do celular.

Quatérnion y é o eixo transverso do celular. Quatérnion z é o eixo sagital do celular.

Quatérnion wé a quantidade escalar de rotação do celular.

TABELA 2 - Valores médios e desvio padrão (DP) índice de correlação cruzada R da relação dos quatérnions e rotações medidos no celular e na central inercial no teste de agachamento simples.

\begin{tabular}{lcccccccc}
\hline & \multicolumn{7}{c}{ Índice de Correlação Cruzada R } & $\begin{array}{c}\text { Coeficiente de } \\
\text { Determinaçáo R }\end{array}$ \\
\hline Variável & $\# \mathbf{1}$ & $\mathbf{\# 2}$ & $\mathbf{\# 3}$ & $\mathbf{\# 4}$ & $\mathbf{\# 5}$ & $\mathbf{M e ́ d i a ( D P )}$ & $\mathbf{M e ́ d i a ( D P )}$ \\
\hline Quatérnion x & 0,51 & 0,55 & 0,51 & 0,52 & 0,55 & $0,53 \pm 0,02$ & $0,28 \pm 0,00$ \\
Quatérnion y & 0,33 & 0,43 & 0,60 & 0,54 & 0,60 & $0,50 \pm 0,12$ & $0,25 \pm 0,01$ \\
Quatérnion z & 0,98 & 0,99 & 0,98 & 0,99 & 0,98 & $0,98 \pm 0,01$ & $0,97 \pm 0,00$ \\
Quatérnion w & 0,98 & 0,99 & 0,98 & 0,99 & 0,98 & $0,98 \pm 0,01$ & $0,97 \pm 0,00$ \\
Adução/abdução & 0,50 & 0,51 & 0,55 & 0,57 & 0,55 & $0,54 \pm 0,03$ & $0,29 \pm 0,00$ \\
Rotação interna/externa & 0,44 & 0,53 & 0,49 & 0,65 & 0,45 & $0,51 \pm 0,09$ & $0,26 \pm 0,01$ \\
Flexão/extensão & 0,95 & 0,97 & 0,95 & 0,99 & 0,94 & $0,96 \pm 0,02$ & $0,92 \pm 0,00$ \\
\hline
\end{tabular}

Os valores estão apresentados por teste (\#1 a \#5) e valor médio. Quatérnion x é a rotação em torno do eixo perpendicular a tela do celular.

Quatérnion y é o eixo transverso do celular. Quatérnion z é o eixo sagital do celular.

Quatérnion wé a quantidade escalar de rotação do celular. 


\section{Discussão}

Este estudo comparou as medidas de quatérnion de sistema de captura de movimento baseado em sensores inerciais e as medidas de quatérnion de um smartphone durante o movimento de agachamento. Para mostrar a possibilidade de tal equivalência, a primeira comparação foi feita em sistema de rotaçáo controlada que é o pêndulo simples. Em seguida, fizemos a comparação das mesmas medidas durante o movimento de agachamento. Nas duas condições, encontramos alta semelhança de medidas de rotação, que foi descrita pela análise dos quatérnions. Estes resultados indicam que é possível usar um smartphone para avaliar a orientação e rotação do corpo durante a prática de atividade física.

Os acelerômetros são transdutores projetados para medir deformaçóes de um sistema massa-mola que são usadas para identificar a aceleração desse sistema ${ }^{5}$. Os acelerômetros podem unidirecionais e os mais típicos medem os componentes da aceleração em três eixos ortogonais ${ }^{14}$. Os acelerômetros são usados como sensores de movimento ${ }^{15}$ e de vibração. Versóes simples dos acelerômetros são usadas nos estudos epidemiológicos para avaliar o nível de prática de atividade física ${ }^{16}$.

Para avaliar a cinemática do movimento humano, os sistemas de captura de movimento convencionais são baseados em filmar o movimento com câmeras de vídeo ou com emissores eletromagnéticos. Estes sistemas de cinemetria ${ }^{1}$ sáo robustos; porém pouco versáteis. Por essa razão, cada vez mais é investido em instrumentação portátil ou vestível ${ }^{17}$. Em contra partida, os dispositivos móveis permitem que esse tipo de ferramenta seja transferido para o contexto real.

O quatérnion é usado para estimar a rotação e orientação de um corpo rígido a partir de informaçóes de sensores magnéticos, campos de aceleração e giroscópio que são sensores mais comuns ${ }^{18}$, além de estar presente nos instrumentos selecionados e a componente que melhor responde as rotaçóes investigadas. No pêndulo simples, a semelhança do padrão do quatérnion $\mathrm{w}$ do smartphone e a central inercial foi alta; pois, a correlação foi muito forte. O pêndulo simples foi escolhido para teste devido à característica de movimento uniaxial, o que permitiu controlar os movimentos propostos. Infere-se que ambas as ferramentas mensuram de forma equivalente o movimento proposto.

As rotaçóes da perna durante o agachamento analisadas pelo sensor inercial e smartphone foram bastante semelhantes. A correlação muito forte do quatérnion W remete a proximidade dos dados avaliados em movimentos dinâmicos. Os sensores inerciais foram adequados para rastrear movimentos do quadril durante o andar, embora tal aplicação possa ser estendida a outras atividades funcionais ${ }^{19}$. Em paralelo, o smartphone pode auxiliar a compreender o fenômeno do movimento e seu padrão. O monitoramento feito pelos sinais do smartphone foi capaz de acompanhar movimentos complexos e dinâmicos como o agachamento. Este resultado potencializa a aplicação do smartphone para avaliar os movimentos do corpo humano em outras tarefas motoras, como a locomoção. $\mathrm{Na}$ avaliação da marcha, a rotaçáo dos membros inferiores pode ser obtida diretamente em sensores inerciais como o Xsens ${ }^{4}$.

Além do nível de atividade física, outras variáveis como postura e equilíbrio ${ }^{15}$ têm sido avaliadas por intermédio desses instrumentos. $\mathrm{O}$ acelerômetro pode ser aplicado para diversos estudos, incluindo balanço postural, déficits de atividade da vida diária, contagens de atividade física que inferem ao sedentarismo. $\mathrm{O}$ smartphone pode estimar diferentes movimentos do corpo humano e permite realizar aplicaçóes de monitoramento da atividade física para a saúde 5 . Para estimar as propriedades do movimento humano, questionamentos como o posicionamento do aparelho também deve ser abordados. O tipo de movimento físico identificável pelos sensores depende de como o smartphone é usado no corpo. Ainda não está claro como os métodos de captura e rastreio do movimento precisam ser desenvolvidos para facilitar o monitoramento não-invasivo ${ }^{5}$.

Benefícios do smartphone mostram resultados promissores e contempla um caminho para o rastreamento do movimento humano. Avanços tecnológicos na acelerometria permitem recolher sinais e superar as limitaçóes destes dispositivos móveis, como tamanho, resistência mecânica e transmissão de dados online. Ferramentas potentes oferecem ganhos, concomitante as dificuldades decorrentes à produção de dados complexos para os quais orientaçóes metodológicas estão em falta ${ }^{20}$, logo, esse avanço deve afrontar com respaldo científico a busca pela aplicabilidade de forma efetiva e sólida. Prontamente, a aplicação clínica, desportiva, recreativa necessita de análises adicionais para 
encontrar parâmetros e garantir o melhor desempenho do movimento ${ }^{4}$.

O sensor de acelerômetro e giroscópio integrado ao smartphone apresenta semelhança significativa com a central inercial. O smartphone mostrou resultados muito semelhantes no balanço do pêndulo bem como os valores de flexão e extensão do quadríceps femoral no agachamento, indicando que ambos os sinais seguem o mesmo padrão de distribuição. Novos experimentos devem explorar ensaios similares para padronizar a interpretação dos resultados disponíveis nos aplicativos. Isso estimulará a discussão de prós e contras desses dispositivos em âmbito profissional, científico ou esportivo.

\title{
Agradecimentos
}

Laboratório de Biomecânica do Centro de Ciências da Saúde e do Esporte da Universidade do Estado de Santa Catarina.

\begin{abstract}
Using the accelerometer and gyroscope in motion monitoring: a comparative evaluation through inertial unit and smartphone

This study compares the acceleration measured by inertial sensors to monitor physical activity. The aim of this study is to compare information about motion measured by inertial sensor (Xsens system) and by a smartphone. Those two instruments measured the rotation of a simple pendulum and the thigh rotation during squats. To compare the time series of rotation of both systems, it was applied the cross correlation analysis. To estimate rotation, it was used the quaternion measurements of each system. For the simple pendulum, rotation described by quaternions of Xsens system and smartphone presented a very strong correlation, with $98 \%$ of similarity. For the squat, the level of correlation was very strong, showing $97 \%$ of similarity. The accelerometer within the smartphone showed signals very similar to the signal measured by the inertial unit.
\end{abstract}

KeYWords: Physical activity; Biomechanics,; Motion analysis; Quaternion.

\section{Referências}

1. Amadio AC, Costa PHL, Sacco ICN, Serrão JC, Araujo RC, Duarte M, Mochizuki L. Introdução à análise do movimento humano: descrição e aplicação dos métodos de medição. Rev Bras Fisioter. 1999;3(2):41-54

2. Cliff DP, Reilly JJ, Okely AD. Methodological considerations in using accelerometers to assess habitual physical activity in children aged 0-5 years. J Sci Med Sport. 2009;12(5):557-67.

3. Jeremy AP, Amick RZ, Thummar T, Rogers ME. Validation of measures from the smartphonesway balance application: a pilot study. Int J Sports Phys Ther. 2014;9(2):135.

4. Guo Y, Zhao G, Liu Q, Mei Z, Ivanov K, Wang L. Balance and knee extensibility evaluation of hemiplegic gait using an inertial body sensor network. Biomed Eng Online. 2013;12(1):83.

5. del Rosario MB, Redmond SJ, Lovell NH. Tracking the evolution of smartphone sensing for monitoring human movement. Sensors (Switzerland). 2015;15(8):18901-33.

6. Jambersi AB, Silva $S$ da. A sutileza dos quatérnions no movimento de rotação de corpos rígidos. Rev Bras Ensino Física. 2016;38(2)

7. Tannous H, Istrate D, Benlarbi-Delai A, Sarrazin J, Gamet D, Ho Ba Tho MC, et al. A new multi-sensor fusion scheme to improve the accuracy of knee flexion kinematics for functional rehabilitation movements. Sensors (Switzerland). 2016;16(11). 
8. Pomares J, Perea I, Garc??a GJ, Jara CA, Corrales JA, Torres F. A multi-sensorial hybrid control for robotic manipulation in human-robot workspaces. Sensors. 2011;11(10):9839-62.

9. Especificação técnica iPhone. 2016. p. Disponível em: https://support.apple.com/kb/SP655? viewlocale=pt_.

10. Especificação técnica SensorLog. 2016. p. Disponível em: https://itunes.apple.com/gb/app/sensorlog/id388014.

11. Especificação técnica Xsens. 2016. p. Disponível em: https://www.xsens.com.

12. SENIAM. Programa de Saúde e Investigação de Saúde e Investigação Biomédica [Internet]. SENIAM (Projeto de padronização de eletromiografia de superfície para a avaliação não invasiva dos músculos). 2016 [citedo 2016 Abr 25]. p. http://seniam.org/]. Disponível em: http://seniam.org/

13. Statistics Corner: a guide to appropriate use of correlation coefficient in medical research. Malawi Med J. 2012;24(3):6971.

14. Vieira LP. Experimentos com o acelerômetro de tablets e smartphones. Dissertação: [Mestrado em Física], Universidade Federal do Rio de Janeiro; 2013.

15. Teruya TT, Matareli BM, Romano FS, Mochizuki L. The effect of a silicone wristband in dynamic balance. Percept Mot Skills. 2013;117(2):353-7.

16. Salvador EP, Ribeiro EH, Garcia LM, Andrade DR, Guimaraes VM, Aoki MS, et al. Interventions for physical activity promotion applied to the primary healthcare settings for people living in regions of low socioeconomic level: study protocol for a non-randomized controlled trial. Arch public Heal. 2014;72(1):8.

17. Mancini M, Salarian A, Carlson-Kuhta P, Zampieri C, King L, Chiari L, et al. ISway: a sensitive, valid and reliable measure of postural control. J Neuroeng Rehabi. 2012;9(1):59.

18. Renaudin V, Combettes C. Magnetic, acceleration fields and gyroscope quaternion (MAGYQ)-based attitude estimation with smartphone sensors for indoor pedestrian navigation. Sensors (Switzerland). 2014;14(12):22864-90.

19. Saber-Sheikh K, Bryant EC, Glazzard C, Hamel A, Lee RYW. Feasibility of using inertial sensors to assess human movement. Man Ther. 2010;15(1):122-5.

20. Cain KL, Sallis JF, Conway TL, Van Dyck D, Calhoon L. Using accelerometers in youth physical activity studies: a review of methods. J Phys Act Heal. 2013;10:437-50.

\begin{tabular}{r|l} 
ENDEREÇO & \\
Beatriz de Araujo Antonio & \\
Escola de Artes, Ciências e Humanidades & Submetido: $16 / 05 / 2017$ \\
Universidade de São Paulo & Revisado: $13 / 11 / 2017$ \\
Rua Arlindo Bettio, 1000 & Aceito: 01/02/2018
\end{tabular}

03828-000 - São Paulo - SP - Brasil

E-mail: filipepitagoras@hotmail.com 